\title{
MARCADORES MOLECULARES RELACIONADOS CON CÁNCER DE PRÓSTATA: 3-NITROTIROSINA Y EXPRESIÓN GÉNICA Y PROTÉICA DE LA Mn-SUPERÓXIDO DISMUTASA (Mn-SOD).
}

\author{
Esaú Floriano-Sánchez', Melchor Castro-Marín² y Noemi Cárdenas-Rodríguez 3 . \\ 'Departamento de Bioquímica y Biología Molecular. Escuela Médico Militar. México, D. F. \\ ${ }^{2}$ Servicio de Urología. Hospital Central Militar. México, D.F. \\ ${ }^{3}$ Laboratorio de Neuroquímica. Instituto Nacional de Pediatría. México, D.F.
}

Resumen.- OBJETIVO: Recientes reportes ubican al cáncer de próstata en el tercer lugar mundial, en México es la principal causa de muerte masculina. En este estudio se evaluaron: marcadores moleculares y un marcador génico en tejidos con cáncer de próstata e hiperplasia prostática benigna (HPB): el gen de la Mn-superóxido dismutasa (Mn-SOD) y su proteína, el antígeno prostático específico (APE) y 3-nitrotirosina (3-NT).

Se evaluó si existe alguna alteración en la expresión de diversos marcadores de estrés oxidativo en cáncer de próstata e hiperplasia prostática benigna.

\section{CORRESPONDENCIA}

Esaú Floriano Sánchez

Escuela Médico Militar

Cerrada de palomas $S / N$

Col. Lomas de San Isidro. (México, D.F.)

floriano_esa@yahoo.com

Trabajo recibido: 16 de enero 2009.
MÉTODOS: Se obtuvieron 80 biopsias. Se estandarizaron las condiciones para amplificar y evaluar la inmunorreactividad del gen de interés (Mn-SOD y 3-NT) y la correlación entre el antígeno prostático específico (APE) y la inmunorreactividad de Mn-SOD en cáncer de próstata e hiperplasia prostática benigna.

RESULTADOS: La sobreexpresión del gen y la inmunorreactividad de Mn-SOD, así como la inmunorreactividad de 3-NT en cáncer de próstata fue mayor con respecto al grupo con hiperplasis prostática benigna. No se observó una correlación entre los niveles de APE y la inmunorreactividad de Mn-SOD.

CONCLUSIONES: Los resultados anteriores sugieren que la determinación de los parámetros evaluados pueden ser utilizados como marcadores tumorales realizando las determinaciones en biopsias de pacientes sospechosos de cáncer de próstata.

Palabras clave: Cáncer de próstata. Hiperplasia prostática benigna. Especies reactivas de oxígeno. Mn-SOD. 3-nitrotirosina. Antígeno prostático específico.

Summary.- OBJECTIVES: Recent reports place prostate cancer (PCa) as third cause of death in the World among males, in Mexico it is the leading cause of male death. In this study, we studied molecular markers and one genetic marker related in tissues with PCa and benign prostatic hyperplasia(BPH) : The Mn-superoxide dismutase (Mn-SOD) gen and protein, prostate specific antigen (PSA) and 3-nitrotyrosine (3-NT). It was evaluated if various markers of oxidative stress show altered expression in prostate cancer and $\mathrm{BPH}$. 
METHODS. 80 biopsies were obtained. The conditions to amplify and evaluate the immunoreactivity of the genes of interest (Mn-SOD and 3-NT) and the correlation between PSA and Mn-SOD immunoreactivity in prostate cancer and benign prostatic hyperplasia were standardized.

RESULTS. Gene overexpression and Mn-SOD and 3-NT immunoreactivity were greater in prostate cancer with respect to the $\mathrm{BPH}$ group. Correlation between levels of PSA and the Mn-SOD immunoreactivity was not observed.

CONCLUSIONS. The above results suggest that the parameters evaluated can be used as tumor markers making the determinations in biopsies of patients suspected of prostate cancer.

Keywords: Prostate cancer. Benign prostatic hyperplasia. Reactive oxygen species. Mn-SOD. 3-nitrotyrosine. Prostate specific antigen.

\section{INTRODUCCIÓN}

El cáncer de próstata (CaP) es la segunda neoplasia más común en los varones después del cáncer de piel y la segunda causa de muerte en hombres por cáncer después del cáncer de pulmón (1). Se atribuyen los numerosos factores al aumento en la incidencia del CaP. Más de 200,000 hombres en los Estados Unidos son diagnosticados anualmente con CaP y 30,000 hombres mueren cada año por esta enfermedad. Después de los 50 años de edad la incidencia de CaP aumenta 3 ó 4 veces cada 10 años (1). Los afroamericanos muestran la tasa de incidencia más elevada de CaP, la incidencia global de CaP en afroamericanos aumento de 124 por 100,000 a 250 por 100,000, un incremento de $102 \%$. Los hispano-americanos presentan una incidencia intermedia de 104 por 100,000. Los países asiáticos, sobre todo Japón y China, presentan una de las tasas más bajas de incidencia y mortalidad por cáncer de próstata en el mundo. La incidencia de mortalidad por CaP en Japón entre 1992 a 1995 fue de 4 por 100,000 (2). En México, el CaP ocupó en 1998 el segundo lugar respecto de la incidencia entre las neoplasias del varón, después del cáncer de piel. En cuanto a la mortalidad, ocupó también el segundo lugar de la totalidad de las neoplasias después del carcinoma cervicouterino (3).

La etiología del CaP no está del todo clara. Hasta el momento, los aspectos que se han considerado como factores de riesgo son: la edad del indi- viduo, la producción y metabolismo de los andrógenos, el área geográfica/etnia, el hábito dietético y la historia familiar.

La hiperplasia prostática benigna (HPB) es el tumor benigno más común en varones y su incidencia está relacionada con la edad. La prevalencia de la HPB es dependiente de la edad, con inicio en su desarrollo después de los 40 años de edad. Para los 60 años de edad, su prevalencia es $50 \%$ y para los 85 años de edad es de $90 \%$.

Al igual que el CaP, está relacionada con la edad del individuo (envejecimiento) y requiere andrógenos (dihidrotestosterona) para desarrollarse y crecer. A diferencia del CaP, la HPB es una lesión benigna que rara vez progresa hacia una neoplasia maligna. Otra diferencia respecto al $\mathrm{CaP}$ es que no existe ninguna evidencia de que HPB sea clonal sólo se han encontrado algunos cambios genéticos que implican estabilidad genómica. En HPB no se detectan grados morfológicos en el núcleo que son característicos en las neoplasias maligna. En la patología hiperplásica con frecuencia presenta crecimientos variables del estroma y se ha sugerido que la secreción de factores de crecimiento por parte del mesénquima podría actuar sobre las células epiteliales adyacentes y contribuir al desarrollo de la hiperplasia de estas últimas (4).

Se estima que la dieta puede contribuir con el $35 \%$ de todos los tumores humanos (5). La evidencia epidemiológica revela consistentemente que una ingestión baja de antioxidantes o un nivel sanguíneo bajo de antioxidantes puede incrementar el riesgo de padecer cáncer.

Recientemente se ha relacionado que el daño oxidativo juega un papel crítico en varias condiciones clínicas como lo son las enfermedades malignas. Las especies reactivas de oxígeno (ERO) pueden causar oxidación del DNA y daño a proteínas, daño a genes, supresores tumorales y un aumento en la expresión de proto-oncogenes. La aparición tardía del carcinoma prostático sugiere que un proceso de múltiples pasos está involucrado en la carcinogénesis y los más razonables candidatos para la formación endógena de genotoxinas en estas etapas tardías de la vida es la acumulación de ERO (6).

Para proteger contra los efectos tóxicos de las ERO y modular sus efectos fisiológicos las células han desarrollado un sistema intrínseco de defensa antioxidante. El sistema antioxidante enzimático es muy complejo, siendo compuesto por pequeñas moléculas de gran peso antioxidante (Vitaminas E, C, A), enzimas antioxidantes primarias (manganeso, cobre, 
zinc superóxido dismutasa, catalasa, glutatión peroxidasa) y enzimas antioxidantes secundarias (glutatión reductasa y glucosa 6 fosfato deshidrogenasa). El óxido nítrico $(\mathrm{NO} \bullet$ ) modula los niveles de ERO en parte por su reacción con el anión superóxido, finalmente las proteínas involucradas en la respuesta al estrés celular también son importantes en la modulación del daño oxidativo; cada componente del sistema antioxidante es específicamente localizado en compartimentos subcelulares (7).

Debido a que el estrés oxidativo está involucrado en la etiopatogenia del cáncer se evaluó la expresión génica y la inmunorreactividad de Mn-SOD así como su relación con el antígeno prostático específico (APE) y la inmunorreactividad de 3-nitrotirosina (3-NT) en CaP y en la HPB.

\section{MATERIALES Y MÉTODOS}

\section{Sujetos de estudio:}

La recolección de muestras se llevó a cabo del mes de diciembre del 2006 a marzo del 2007, seleccionando las muestras de los pacientes registrados en la libreta de cirugía del servicio de urología del Hospital Central Militar y que cumplieron con los criterios de inclusión, exclusión y eliminación;

\section{Criterios de inclusión:}

a) Pacientes con diagnóstico de CaP con síndrome obstructivo urinario bajo en las vías urinarias.

b) Pacientes con diagnóstico de HPB con indicación de resección transuretral prostática (RTUP) y prostatectomía radical;

\section{Criterios de exclusión:}

a) Pacientes que no autorizan participar en el procedimiento.

b) Pacientes que no sean candidatos a RTUP.

\section{Criterios de eliminación:}

a) Pacientes cuyo RNA extraído y purificado presentaba una concentración y pureza inadecuada para el estudio.

b) Tejido que en el traslado sufrió desnaturalización del material genético.

La cantidad de tejido necesaria para la Investigación fue de 1 hasta $5 \mathrm{~g}$, la obtención de las muestras se hizo inmediatamente después de la rea- lización de la intervención quirúrgica, el tiempo de traslado fue de una hora como máximo; desde la obtención de la muestra hasta su traslado al laboratorio de Biología Molecular de la Escuela Médico Militar, donde se mantuvieron a una temperatura de $-70^{\circ} \mathrm{C}$ en el ultracongelador Revco® (Legaci ULT21 186 3-35 Dupont SVVA Refrigerants) hasta el procesamiento de la extracción de RNA.

\section{Extracción y cuantificación de RNA}

EI RNA total fue extraído con el kit de extracción de RNA total usando el SV Total RNA Isolation System (Promega, Madison, WI, USA), se tomaron $150 \mathrm{mg}$ de cada una de las muestras con técnica estéril, evitando contaminación de RNAsas, homogenizándolas con un politrón (Omni $\mu \mathrm{H}$ International), siguiendo las indicaciones del proveedor, por último a la elución final de RNA total se les agregó $2 \mu \mathrm{L}$ de inhibidor de RNAasas y se guardaron a $-83^{\circ} \mathrm{C}$ para posteriores análisis. La verificación de la integridad del RNA total se realizó por medio de electroforesis en un gel de agarosa al $2 \%$, colocando $6 \mu \mathrm{l}$ del RNA total extraído por muestra y se visualizaron las bandas con luz UV (EDAS 290 KODAK). La cuantificación del RNA total se llevo a cabo por medio de fluorescencia con SYBR Green, con el multidetector Synergy HT-I (BIO-TEK Instruments, Inc. Higland Park, Vermont, USA).

\section{Estandarización de la técnica de RT-PCR en tiempo real}

Para el desarrollo de la RT- PCR en tiempo real se diseñaron oligonucleótidos específicos (primers) del gen de interés (Mn-SOD) y para los genes de referencia (candidatos endógenos): subunidad ribosomal 18s, gliceraldehido-3 fosfato deshidrogenasa (GAPDH), glucosa 6 fosfato deshidrogenada (G6PDH) y ß-actina (BACT); los genes endógenos se validaron con el software BestKeeper y se tomo el más estable como control endógeno de la carga de RNA total. Las secuencias fueron obtenidas del GenBankTM (Tabla I). La búsqueda de las secuencias se realizó en (http://www.ncbi.nlm.nih.gov/BLAST/) para confirmar su especificidad. Los primers sintetizados (Invitrogen, Carlsbad, CA) fueron los siguientes: gen de interés $\mathrm{Mn}$-SOD; genes candidatos endógenos: BACT, 18s, GAPDH y el gen G6PDH, los cuales fueron optimizados a una temperatura de $57^{\circ} \mathrm{C}$ (Tabla I).

Las condiciones para la RT-PCR fueron optimizadas con un termociclador de gradiente (gradient Px2 Thermal Cycler Hybaid, Franklin, MA) utilizando el kit SuperScriptTM III Platinum $®$ SYBR® One-Step qRT-PCR Kit with ROX (Invitrogen, Carlsbad, CA). La 
secuencias tomadas corresponde a los registros en el GenBankTM bajo el siguiente número de accesiones: Mn-SOD, NM_017051; BACT, NM_001101 ; GAPDH, NM_002046; subunidad 18s, X03205 y G6PDH, NM_004285. Los productos de amplificación por RT-PĆR fueron visualizados por electroforesis en un gel de agarosa al $2 \%$ y estudiados con el sistema de análisis de electroforesis EDAS 290, (KODAK, New Haven, CT). Los resultados obtenidos de las amplificaciones como son las condiciones de temperatura, concentraciones de primers, dNTPs (nucleótidos) y volúmenes, fueron transferidos al protocolo de amplificación en tiempo real con el sistema de detección Rotor gene 6.0 (Corbett Life Science, Sidney City, Australia).

\section{Protocolo de RT-PCR en tiempo real Rotor- Gene 3000}

Las reacciones de la RT-PCR en tiempo real se realizaron con 10ng de RNA extraído de los tejidos estudiados; las diluciones para la validación del control interno, cuantificación absoluta y la de- terminación de las eficiencias de todos los primers, se realizaron usando el kit SuperScript ${ }^{T M}$ III Platinum ${ }^{\circledR}$ SYBR尺 One-Step qRT-PCR Kit with ROX (Invitrogen, Carlsbad, CA), el cual es una mezcla de reacción que contiene un complejo de Taq DNA polimerasa (Platinum) con anticuerpo, el cual inhibe la actividad a temperatura ambiente y dicho anticuerpo es desnaturalizado en la fase inicial del ciclo de la PCR $\left(95^{\circ} \mathrm{C}\right.$ a los 5 minutos). Los siguientes componentes de la reacción fueron preparados a una concentración final: de la mezcla de reacción de $6.25 \mu$ l 2X SYBR® Green I (un amortiguador contenido en SYBR® Green I dye) $0.4 \mathrm{mM}$ por cada dNTP y $6 \mathrm{mM} \mathrm{MgSO}_{4}$, (ROX Reference Dye) el cual es un estabilizador del amortiguador (1X), $4 \mu$ l RNA total, $0.25 \mu l$ SYBR尺 Green One Step Enzime Mix (contenida en SuperScript ${ }^{T M}$ III RT Platinum ${ }^{\circledR}$ Taq DNA polymerase, y Inhibidor de RNasa (Recombinante de Ribonucleasa), $0.4 \mu l$ $\mathrm{MgCl}_{2}(50 \mathrm{mM}), 0.4 \mu \mathrm{l}$ del primer forward $(0.4 \mu \mathrm{M})$, $0.4 \mu \mathrm{l}$ del primer reverso $(0.4 \mu \mathrm{M})$, y $0.8 \mu \mathrm{l}$ de agua libre de RNAsas (12.5 $\mathrm{l}$ de la mezcla reacción final), y llevados a tubos $100 \mu$ l. La reacción se llevó a cabo inicialmente a $52^{\circ} \mathrm{C}$ por 10 minutos para la síntesis

TABLA I. SECUENCIA DE LOS PRIMERS QUE SE USARON EN LA RT-PCR, POSICIÓN, SECUENCIA, TAMAÑO DEL AMPLICÓN, LA ACCESIÓN (GENBANK'TM) Y EFICIENCIA.

\begin{tabular}{|c|c|l|c|c|c|}
\hline \multicolumn{5}{|c|}{ Iniciadores (primers) utilizados en la RT-PCR en tiempo real } \\
\hline Blancos & Posiciones & \multicolumn{1}{|c|}{ Secuencia del nucleótido } & Longitud del & Secuencia del & Eficiencia de la \\
ampliación & GenBank ${ }^{\text {TM }}$ & amplificación \\
\hline BACT & $1031-1173$ & F:CTGGCACCCAGCACAATG & $143 \mathrm{pb}$ & NM_001101 & 2.60 \\
\hline $18 S$ & $1601-1713$ & F: GTAACCCGTTGAACCCCATT & $151 \mathrm{pb}$ & X03205 & 2.27 \\
\hline MnSOD & $301-352$ & F:TGTGTCTGTGGGAGTCCAAG & $151 \mathrm{pb}$ & NM_017051 & 2.00 \\
\hline GAPDH & $446-620$ & F: GAGCCAAAAGGGTCATCATCTC & $175 \mathrm{pb}$ & NM_002046 & 2.04 \\
\hline G6PDH & $1777-1935$ & F: CCTGGAGGAGCTGAGAATGG & $159 \mathrm{pb}$ & NM_004285 & 2.66 \\
\hline
\end{tabular}


de DNA y $94^{\circ} \mathrm{C}$ por 5 minutos para la el inicio de la actividad de la Taq polimerasa (Platinum Taq) y la predesnaturalización del híbrido RNA-cDNA, seguido de los ciclo de amplificación de la PCR con 40 ciclos, consistente cada ciclo de $95^{\circ} \mathrm{C}$ por 20 segundos (s), $57^{\circ} \mathrm{C}$ por $20 \mathrm{~s}, 72^{\circ} \mathrm{C}$ por $20 \mathrm{~s}$, el cual tuvo tres lecturas de puntos de fluorescencia a 57,72 y $83^{\circ} \mathrm{C}$, respectivamente. El paso de la curva melting tuvo un intervalo de temperatura de $60-98^{\circ} \mathrm{C}$ con un incremento de temperatura de $0.1^{\circ} \mathrm{C}$ por segundo y finalmente un paso a $40^{\circ} \mathrm{C}$. Para la cuantificación absoluta fue necesario determinar el ciclo umbral $\left(C_{t}\right)$ para cada trascrito. La expresión tanto de los genes candidatos endógenos como del gen de interés $(\mathrm{Mn}$ SOD); fueron cuantificados individualmente con el modelo estadístico BestKeeper Gene para la validación de los endógenos y con el modelo estadístico (RESTC) para el análisis de las eficiencias y la cuantificación relativa de los genes endógenos y Mn-SOD.

\section{Inmunohistoquímica}

Por microscopía de luz, las muestras de tejidos de $\mathrm{CaP}$ y de HPB fueron fijadas por inmersión en formalina $(\mathrm{pH}=7.4)$ y se embebieron en parafina. Para el análisis histológico, secciones de tejido $(3 \mu \mathrm{m})$ fueron teñidas con hematoxilina y eosina (H\&E). Las secciones de tejido fueron teñidas con ácido periódico de Shiff`s (PAS) para mostrar los polisacáridos, mucopolisacáridos y glicoproteínas de la membrana celular.

Los cortes fueron incubados con ácido peryódico durante 5 min y lavados con agua destilada. Los cortes fueron incubados con el reactivo de Schiff's durante 5 min y contrateñidos con hematoxilina por $30 \mathrm{~s}$. El perfil histológico de 5 campos seleccionados aleatoriamente fueron registrados utilizando el software KS-300 (Carl Zeiss, Jena, Germany). El porcentaje de área dañada con alteraciones histopatológicas fue obtenido (magnificación 400x). Para la inmunohistoquímica las secciones de tejido $(3 \mu \mathrm{m})$ se desparafinaron y se calentaron para desenmascarar los sitios antigénicos; la actividad endógena de la peroxidasa fue bloqueada con $0.03 \%$ de $\mathrm{H}_{2} \mathrm{O}_{2}$ en metanol absoluto. Las secciones de tejido fueron incubadas toda la noche a $4^{\circ} \mathrm{C}$ a una dilución 1:100 de anticuerpo monoclonal contra Mn-SOD o contra 3-NT según el caso, en solución TRIS Se removió el anticuerpo primario y se realizaron dos lavados repetitivos con TRIS, los cortes fueron incubados con una dilución 1:500 de anticuerpo policlonal de conejo como anticuerpo secundario y se realizaron dos lavados repetitivos con TRIS. Los anticuerpos unidos se detectaron con el complejo avidina-biotina (ABC-kit Vectatastain) y la diaminobenzidina como sustrato. Después de lavar repetidamente con TRIS los cortes fueron contrateñidos con hematoxilina. Todos los cortes fueron incubados bajo las mismas condiciones con la misma concentración de anticuerpo y en la misma corrida, por lo tanto, la inmunotinción fue comparable. Todos los especimenes fueron examinados por el microscopio de luz Axiovert 200M (Carl Zeiss, Jena, Germany). Para el análisis morfométrico automatizado, el porcentaje de células positivas (color marrón) se determinaron con un analizador de imágenes computarizado KS-300 3.0 (Carl Zeiss, Jena, Germany). Este equipo detecta automáticamente las células positivas determinando su porcentaje por campo. Cinco campos aleatorios fueron estudiados a una magnificación de 100 (área total 1,584,000 $\mu^{2}$ ). Los resultados fueron expresados como porcentaje.

\section{Análisis de datos}

Los datos de las eficiencias y de la cuantificación absoluta $\left(C_{T}\right)$ de los genes fueron analizados usando el software Rotor-Gene 6.0 (Corbett Life Science, Sidney City, Australia). La determinación de los $C_{T}$ fue realizada con el software antes mencionado; los datos fueron graficados con el mismo y exportados a una tabla del Excel dando regresiones lineales similares para las eficiencias de los primers, donde se compara la concentración logarítmica contra el $\left(C_{T}\right)$, y los datos de las curvas de calibración para Mn-SOD y GAPDH fueron analizados por el mismo software antes mencionado. Para la determinación de la pendiente y los coeficientes correlación de Pearson se utilizó una tabla de Excel. Para la validación se utilizó el modelo estadístico BestKeeper Gene.

\section{Análisis estadístico de datos}

Los resultados de expresión génica se analizaron en una tabla de Excel donde previamente se importaran los $C_{T}$ del software Rotor gene 6.0, y se utilizará el software PRISMA tomando las relaciones representativas de HPB y las muestras con CaP.

Se utilizo la Prueba " $t$ " de Student para compararlos cambios en la regulación génica, la inmunorreactividad de 3-NT y la inmunorreactividad del gen de $\mathrm{Mn}$-SOD en ambos tejidos. Se considero una $\mathrm{p}<$ 0.05 como diferencia estadísticamente significativa Para la aplicación de las pruebas estadísticas se utilizó el programa Graph Prisma versión 3.32.

\section{RESULTADOS}

\section{a. Expresión génica de MnSOD}

Del total de los 80 pacientes de los cuales se obtuvieron las muestras, 27 pacientes (33.7 \%) tenían diagnóstico establecido de $\mathrm{CaP}$, mientras que 
53 pacientes (66.2\%) tuvieron diagnóstico establecido de HPB.

Para poder determinar el endógeno adecuado y el más estable se realizó RT-PCR en tiempo real por triplicado de cada una de las muestras de CaP y se compararon tomando las $C_{\mathrm{T}}$ (la media del triplicado de cada muestra); estos datos se exportaron al software REST (Figura 2).

Después de exportar y analizar los datos se tomó como gen endógeno a GAPDH, el cual presentó una estabilidad superior a los otros tres, con una $p<0.005$ y un coeficiente de correlación de Pearson de 0.953 con el gen de interés Mn-SOD (Tabla II).

Las copias absolutas de mRNA de Mn-SOD de CaP y HPB se compararon con la prueba " $t$ " con una diferencia estadística de $\mathrm{p}=0.0002$; la media de copias absolutas para CaP fue de $765.7 \pm 185.2$; La media de HPB fue de $242.8 \pm$ de 18.43. Por lo que después del análisis de los resultados de estos datos de expresión encontramos que hay 3.15 veces mas de sobreexpresión génica de Mn-SOD en CaP comparado con HPB (Figura 2).

\section{b. Correlación de niveles de APE vs inmunorreactivi- dad del tejido con CaP}

Mediante la prueba de correlación de Pear- son, no se encontró correlación entre los niveles de expresión de la Mn-SOD y los niveles séricos de APE (Figura 3).

\section{c. Inmunorreactividad de MnSOD}

El porcentaje de área inmunoreactiva a $\mathrm{Mn}$ SOD fueron $24.83 \pm 10.5 \%$ y de $14.73 \pm 8.7 \%$ en $\mathrm{CaP}$ y HPB, respectivamente (Figura 4).

\section{d. Inmunorreactividad de 3-NT}

El porcentaje de área inmunoreactiva a 3-NT en fueron de $25.78 \pm 15.37 \%$ y de $4.43 \pm 2.2 \%$ en $\mathrm{CaP}$ y en HPB, respectivamente (Figuras 5 y 6 ).

\section{DISCUSIÓN}

El daño oxidativo causado por las ERO están implicados en la carcinogénesis prostática. La evidencia de la literatura indica que Mn-SOD funciona como un supresor tumoral, posiblemente por la modulación apoptótica y del crecimiento y proliferación celular, además de una relación entre el CaP y ciertos polimorfismos. En nuestro estudio encontramos un aumento en esta enzima, en forma significativa. También se observó un incremento en el porcentaje de área marcada en forma significativa en tejido tumoral. Sin embargo, en varios estudios se ha observado una expresión mayor de Mn-SOD en tejido de cáncer en estadios avanzados (5).

\section{TABLA II. DETERMINACIÓN DEL GEN ENDÓGENO MÁS ESTABLE, LOS DATOS (CT) ANALIZADO POR EL MO-} DELO ESTADÍSTICO BESTKEEPER GENE.

\begin{tabular}{|c|c|c|c|c|}
\hline & BACT & $18 s$ & GAPDH & G6PDH \\
& vs & vs & vs \\
& BK & BK & BK & BK \\
\hline Coeficiente de correlación [r] & 0.940 & 0.868 & 0.953 & 0.070 \\
\hline Intercepción [CP] & -9.162 & -4.016 & -1.910 & 19.981 \\
\hline Pendiente & 1.522 & 1.083 & 1.100 & 0.044 \\
\hline Desviación estandard[SD] & +-0.565 & +-0.636 & +-0.436 & +-0.643 \\
\hline Valor- p & 0.007 & 0.025 & 0.005 & 0.895 \\
\hline Poder [x- veces] & 2.87 & 2.12 & 2.14 & 1.03 \\
\hline
\end{tabular}


En este estudio encontramos que el gen que codifica para Mn-SOD se encuentra expresado 3.15 veces más en tejido de CaP que en HPB. Por otra parte se puede postular que esta actividad aumentada esté provocando la alteración de funciones celulares produciendo respuestas como proliferación celular y muerte celular programada (apoptosis), pudiendo ser una de las causas de la evolución de la enfermedad, incluso del grado de malignidad. Hay datos que nos muestran que la expresión de las enzimas antioxidantes, como Mn-SOD y glutatión-S-transferasa (GST), es posible que estas enzimas o su producto génico (mRNA) puedan servir como marcadores tempranos de cambios premalignos que tengan un riesgo alto de cáncer prostático invasivo. En otros trabajos previos hay correlación con nuestro estudio en los que se ha encontrado que la enzima Mn-SOD esta aumentada en diversos tipos de cáncer (6-11).

Las observaciones anteriores sugieren que el desarrollo selectivo de inhibidores para la Mn-SOD serán de gran interés como potencial estrategia terapéutica para ciertos cánceres, incluso el de próstata (12).

Es muy probable que cambios epigenéticos o alguna mutación en el gen de Mn-SOD sean la causa del alto nivel que encontramos en la expresión de Mn-SOD en el CaP y que esto a su vez sea la causa de los altos niveles de esta proteína encontrados en la mitocondria de las células con $\mathrm{CaP}$, hallazgo encontrado en otro de nuestros estudios (datos no mostrados). Lo anterior podría tener efectos potenciales en la supervivencia y proliferación de las células tumorales, hecho que se ha encontrado en otros tumores que se comportan en forma agresiva y con mal pronóstico para el paciente. Nuestros resultados demuestran que la sobreexpresión génica de esta enzima también juega un papel importante, ya que este producto génico es necesario para la codificación y traducción de dicha proteína.

Por otro lado, la inflamación crónica se ha relacionado con la incidencia de muchos cánceres, incluido el de la próstata (13-15). La inflamación puede dar lugar a la persistencia de estrés oxidativo en las células cancerosas y las especies reactivas del oxígeno pueden representar para ellas una ventaja en la supervivencia (6).

El antígeno prostático específico (APE), es una sustancia proteica exclusiva de la próstata y cuya función es la disolución del coágulo seminal. Los niveles normales en sangre de APE en los varones sanos son muy bajos, del orden de millones de veces menos que el semen, y se elevan en diversas enfermedades de la próstata $(16,17)$, en este estudio encontramos que los niveles de APE en nuestros pacientes las concentraciones fueron de 0.01 a 41.26 .

La concentraciones séricas del APE son directamente proporcionales al volumen de la zona transicional, zona histológicamente bien definida y que en el CaP se encuentra en la mayoría de los casos aumentada, por lo que de acuerdo a este estudio, encontramos que los datos encontrados en este estudio son consistentes con la literatura, ya que ciertos autores describen aumento en la expresión de $\mathrm{Mn}$ SOD en algunos tumores $(8,10)$ y por otro lado los niveles de APE por arriba de 4 e incluso niveles inferiores son un marcador de riesgo de CaP. Los niveles

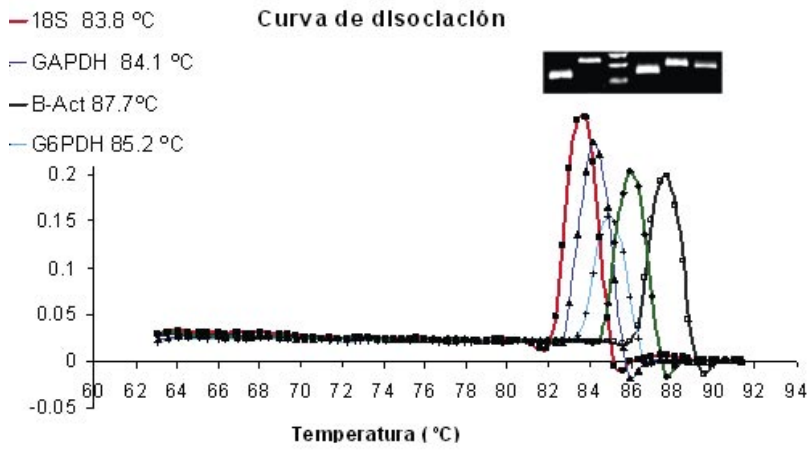

FIGURA 1. Curvas de disociación de los productos de los genes analizados. Con la metodología del SYBR Green I se representa en la curva melting de cada amplicon analizado, inicialmente usando el software Rotor-Gene 6.0 (Corbett Life Science, Sidney City, Australia).

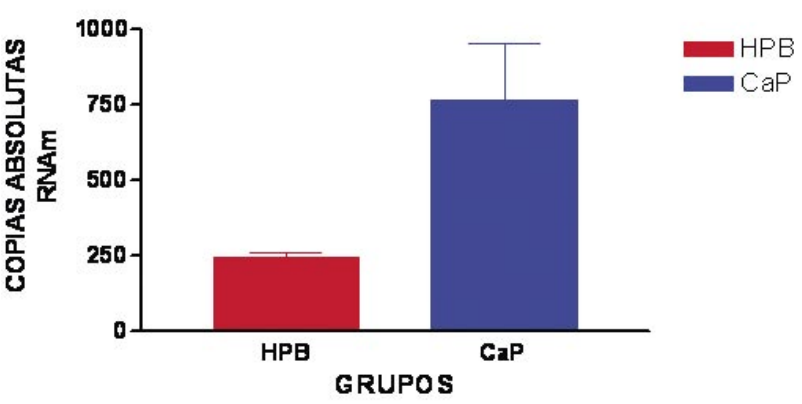

FIGURA 2. Expresión génica de Mn-SOD. Se compararon las copias absolutas de Mn-SOD en ambos grupos de CaP y HPB ( $p=0.0002$ ), en el eje de las $Y$ se muestra el numero de copias absolutas de ambos grupos. Para HPB (SEM=18.43), para CaP (SEM=185). 


\section{Correlación del APE y densitometrías de Mn-SOD}

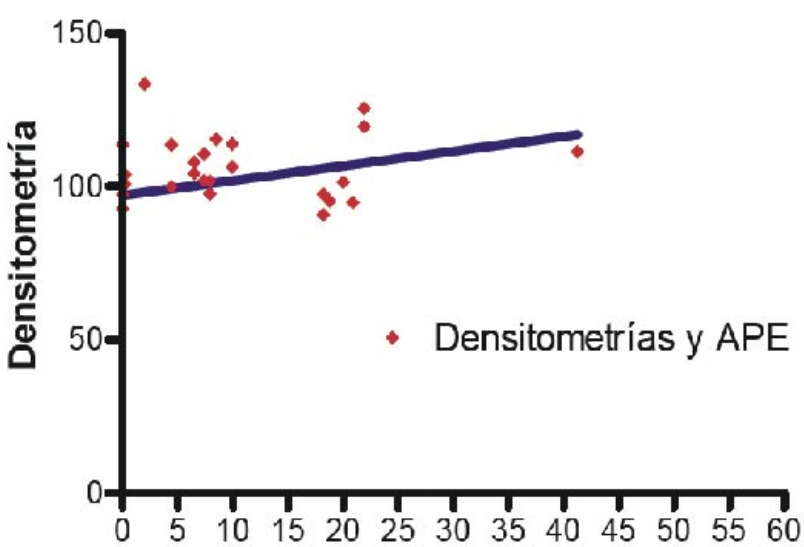

FIGURA 3. Correlacion entre APEs y densitometrías. En " $y$ " valores densitométricos de la inmunoreactividad de la Mn-SOD en tejidos de CaP, en " $x$ " valores de APE de pacientes con CaP. La grafica muestra que los datos de APE tienen una baja correlación con los valores densitométricos de la inmunoreactividad de Mn-SOD en pacientes con CaP. $\mathrm{N}=80$, índice de correlación de Pearson de $0.04182, p=0.4163$.

de APE de 10, indican riesgo alto de incidencia de $\mathrm{CaP}$, encontrando sin embargo, que la medición de la densidad de $A P E$, no es un importante predictor del CaP por lo que todavía se desconoce si el tamiz con APE reduce significativamente la mortalidad de CaP (18).

La Mn-SOD tiene efectos reguladores sobre el potencial redox de la célula, influye en el crecimiento celular y en la expresión génica. Nuestros resultados sugieren un aumento en el porcentaje del área marcada en tejido glandular, sugiriendo que la Mn-SOD puede estar sobre-expresándose en forma más difusa en diferentes tejidos, tanto de áreas de estroma como de áreas de tejido glandular de CaP.

Nuestros resultados sugieren que hay una alteración en el balance pro-oxidante-antioxidante en el CaP, este desbalance, se sabe que altera los procesos oxidorreducción celulares, el crecimiento, proliferación y ciclos celulares, ya que se sabe que ciertos radicales libres median la activación de vías de transducción celular, de factores de trascripción como son Fos, Jun, y el factor nuclear KB y un aumento en la actividad mitocondrial en dichas células (19). Por otro lado se ha demostrado que hay diferencias en la morfología, crecimiento y tumorogenicidad celular de acuerdo con el incremento de la Mn-SOD tanto a nivel génico como a nivel de proteína. Nuestros resultados sugirieron que más allá de la proteína $M n-S O D$, el gen sobreexpresado altera la situación intracelular en la mitocondria o en el número mitocondrial.

En este estudio observamos un incremento en la inmunorreactividad de 3-NT en CaP en compara-
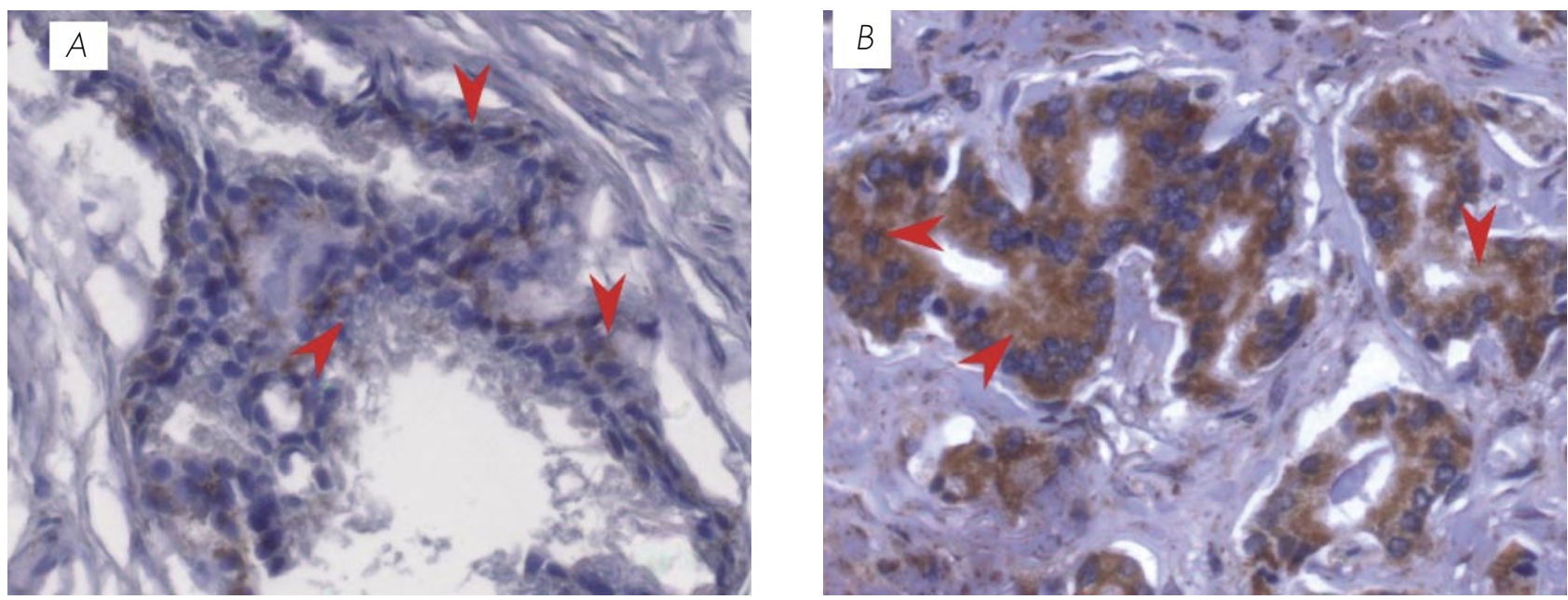

FIGURA 4. Se muestra la localización inmunohistoquímica de "MnSOD", en tejido glandular de HPB y de cáncer de próstata. Las imágenes son cortes histológicos donde se observa la marca de MnSOD, en (A) tejido glandular de "HPB", en (B) tejido glandular de cáncer de próstata. Las flechas indican la inmunorreactividad positiva en ambos tejidos. Se observa disminución evidente de la intensidad de la marca en "A"; tanto en forma difusa como densitométricamente ( $p=0.0001$, SEM). Las figuras son representativas ( $N=80$ ), (amplificación del campo $\times 400$ ). 


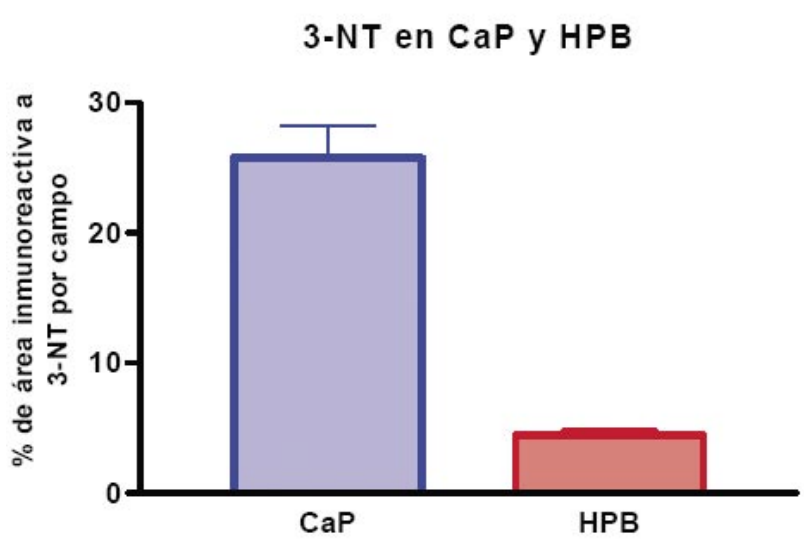

FIGURA 5. Determinación de 3-NT en CaP y HPB. En el eje de las ordenadas se representan los valores del porcentaje de área inmunoreactiva a 3-NT por campo en tejidos de CaP e HPB, en el eje de las abscisas se representan los grupos de estudio (CaP e HPB). En la gráfica se muestra que el área marcada (inmunoreactiva) para 3-NT (3-nitrotirosina) es mucho mayor en tejidos con CaP comparados con tejidos con HPB. $N=80$, CaP y $N=30$ de HPB, $p<0.001$. Los valores se representan como la media \pm desviación estándar.

ción con la HPB. Ya se han determinado alteraciones tanto para $\mathrm{NO}$ - (especie reactiva relacionada con la nitración de proteínas) como sus metabolitos, en cáncer de pulmón y demostraron que $\mathrm{NO} \bullet$, nitritos y nitrotirosina están incrementados en pacientes con cáncer de pulmón, este dato es consistente con lo encontrado en nuestro estudio (20).

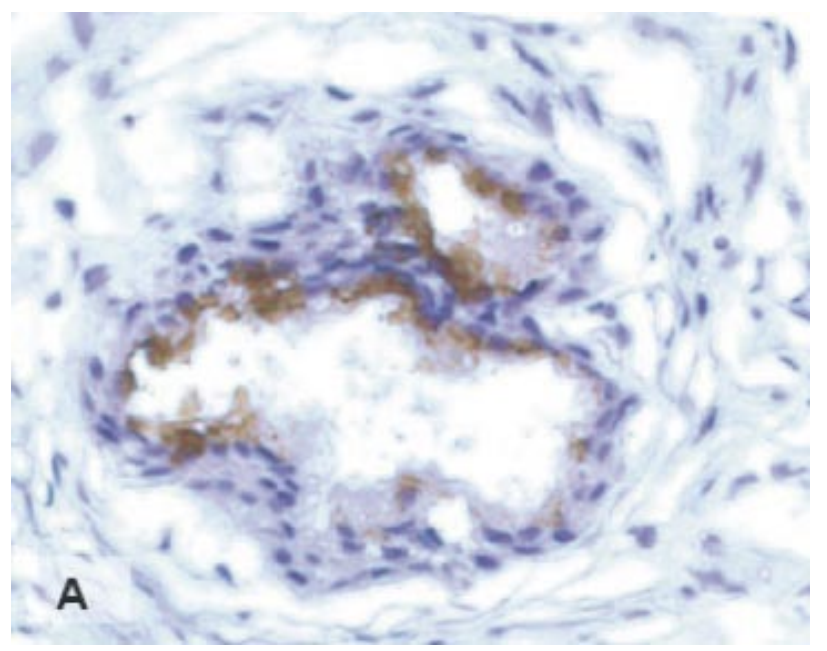

Por otra parte, en el cáncer, se ha encontrado que la expresión de las tres isoformas de las sintasas de NO• (inducible: iNOS, endotelial: eNOS y neuronal: $\mathrm{nNOS}$ ) están aumentadas teniendo una función importante en el desarrollo de tumores y de la angiogénesis. Estas sintasas no han sido suficientemente estudiadas en el $\mathrm{CaP}$, el dato indica que las concentraciones de estas enzimas son bajas en el tejido benigno de este órgano y ellos concluyen que la expresión de iNOS epitelial puede ser usada como un marcador especifico de $\mathrm{CaP}$, ya que el $\mathrm{NO} \bullet$ en parte, tiene una función importante en el desarrollo de la enfermedad (21).

La expresión de la eNOS podría tener una función importante en el proceso carcinogénico del tejido prostático. La producción de $\mathrm{NO} \bullet$ a expensas de esta enzima, puede promover la progresión a cáncer, al regular la proliferación selectiva de las células tumorales, mediante el estimulo angiogénico en el órgano (22).

Estos resultados apoyan lo encontrado en nuestro estudio, se observa niveles de concentración diferencial, ya que hay mayor nitración en las células endoteliales que en otras áreas del tejido canceroso y la diferencia es mayor entre tejidos con HPB comparados con los tejidos con $\mathrm{CaP}$, la concentración de 3-NT es 6-8 veces mas en CaP.

Los resultados que se presentan en este estudio sugieren que el gen de expresión Mn-SOD puede tener efecto sobre ambos procesos tanto en la función mitocondrial, como en el crecimiento de células tumo-

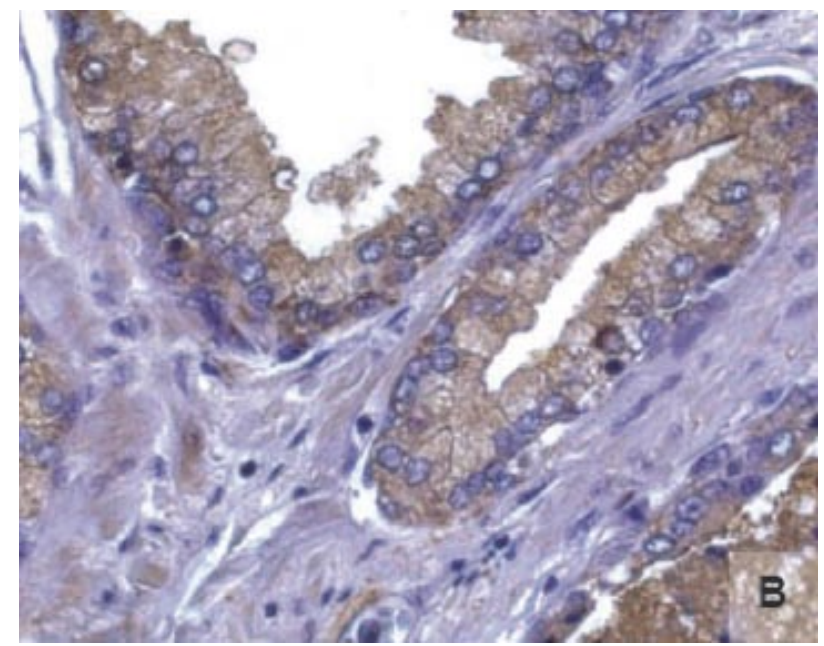

FIGURA 6. Localización de 3-NT por inmunohistoquímica. Imágenes representativas de la expresión de 3-NT en cortes de tejido humano con diagnóstico histopatológico de HPB y CaP. (A) HPB con expresión de 3-NT. (B) CaP con expresión de 3-NT. El tejido glandular de próstata con HPB y CaP muestra inmunotinción positiva para 3-NT (color café o marrón). Las microfotografías son representativas(n=3) y el campo representa 40x. 
rales sin que exista una correlación con los niveles del APE y que la NOS podría estar involucrada en el crecimiento tumoral del $\mathrm{CaP}$, pues ya se ha encontrado que la iNOS esta involucrada en estimulación de la angiogénesis y por las mutaciones incrementadas en el ADN a través de la acción directa de radicales libres (23).

\section{BIBLIOGRAFÍA y LECTURAS RECOMENDADAS (*lectura de interés $y^{* *}$ lectura fundamental)}

*1. De Torres Ramírez I. Late pathology advances in the diagnosis and prognosis of prostate cancer. Arch. Esp. Urol. 2006; 59:1089-110.

2. Ahmed MM, Lee CT, Oesterling JE. Current trend in the epidemiology of prostatic disease: benign hyperplasia and adenocarcinoma. Boca Raton, Florid. CRC Press, 1997.

3. Instituto Nacional de Cancerología, Manual de Oncología, Editorial McGrawHill, 2003.

*4. De Marzo AM, Nelson WG, Meeker AK, et al. Stem cell features of benign and malignant prostate aepithelial cells. J. Urol. 1998; 160:2381-92.

**5. Ballesteros Sampol JJ. Ageing, diet, and prostatic cancer. Arch. Esp. Urol. 2002; 55:225-32.

**6. López Laur JD, Abud M, López Fontana C, Silva J, Cisella Y, Pérez Elizalde R, et al. Antioxidant power and cellular damage in prostate cancer. Arch. Esp. Urol. 2008; 61:563-69.

*7. Oberley TD. Oxidative Damage and Cancer. AJP. 2002; 160:403-8.

8. Kahlos K, Anttila S, Asikainen T, et al. Manganese superoxide dismutase in healthy human pleural mesothelium and in malignant pleural mesothelioma. Am. J. Respir. Cell Mol. Biol. 1998; 18:570-80.

9. Church SL, Grant JW, Ridnour LA, et al. Increased manganese superoxide dismutase expression suppresses the malignant phenotype of human melanoma cells. Proc. Natl. Acad. Sci. 1993; 90:3113-7.

10. Izutani R, Asano S, Imano M, et al. Expression of manganese superoxide dismutase in esophageal and gastric cancers. J. Gastroenterol. 1998; 33:816-22.

11. Nakata T, Suzuki K, Fujii J, et al. High expression of manganese superoxide dismutase in 7,12 dimet hylbenz[a]anthracene-induced ovarian cancer and increased serum levels in the tumor-bearing rats. Carcinogenesis, 1992; 13:1941-3.
*12. Bostwick DG, Alexander EE, Singh R, et al. Antioxidant enzyme expression and reactive oxygen species damage in prostatic intraepithelial neoplasia and cancer. Cancer, 2000; 89:123-34.

**13. Gonzalo ML, Isaacs WB. Molecular pathways to prostate cancer. J. Urol. 2003; 170:2444-6.

14. Catalona W, Partin A, Slawin K, et al. Use of the percentage of free prostate-specific antigen to enhance differentiation of prostate cancer from benign prostatic disease: a prospective multicenter clinical trial. JAMA 1998; 279:1542-7.

15. VanLersel MP, Witjes WP, De la Rosette JJ, et al. Prostate specific antigen density: correlation with histological diagnosis of prostate cancer, benign prostatic hyperplasia and prostatitis. Br. J. Urol. 1995; 76:47-53.

16. Yang QF, Sakurai T, Shan L, et al. Novel polymorphisms of prostate-specific antigen (PSA) gene associated with PSA mRNA expression in breast cancer. Journal of human genetic, 2000; 45:363-6.

17. Torrijos FS, Alcina EL, Escudero JU, Deltoro MF, García AM, Romaguera AR, et al. Fast test serum PSA determination (Sd Bioline PSA). Arch. Esp. Urol. 2008; 61:685-90.

18. Thompson IM, Pauler DK, Goodman PJ, et al. Prevalence of prostate cancer among men with a prostate-specific antigen level $<$ or $=4.0 \mathrm{ng}$ per milliliter. N. Engl. J. Med. 2004; 350:2239-46.

19. Hu Y, Rosen DG, Zhou Y, et al. Mitochondrial manganese-superoxide dismutase expression in ovarian cancer role in cell proliferation and response to oxidative stress. The J. Biol. Chem. 2005; 280:39485-92.

20. Masri FA, Comhair SA, Koeck T, et al. Abnormalities in nitric oxide and its derivatives in lung cancer. Am. J. Resp. Crit. Care Med. 2005; 172:597-5.

*21. Klotz T, Bloch W, Volberg C, et al. Selective expression of inducible nitric oxide synthase in human prostate carcinoma. Cancer. 1998; 82:1897-1903.

22. Marangoni K, Neves AF, Cardoso AM, et al. The endothelial nitric oxide synthase Glu-298-Asp polymorphism and its mRNA expression in the peripheral blood of patients with prostate cancer and benign prostatic hyperplasia. Cancer Detect Prev. 2006; 30:7-13.

23. Baltaci S, Orhan D, Gögüs C, et al. Inducible nitric oxide synthase expression in benign prostatic hyperplasia, low and high grade prostatic intraepithelial neoplasia and prostatic carcinoma. BJU Int. 2001; 88:100-3. 